\title{
Induced Periapical Bleeding in Regeneration of Immature Permanent Teeth with Necrosis: A Systematic Review
}

\author{
Ammar S Al Shamrani
}

\begin{abstract}
Background and objectives: Regenerative treatment of immature teeth leads to continued root maturation thereby restores the physiologically functional pulp-dentin complex. The present systematic review is conducted to evaluate the efficacy of regenerative endodontic treatment by induced periapical bleeding (IPB) in the regeneration of immature necrosed permanent teeth with an open apex.

Materials and methods: This review was conducted in adherence to PRISMA standards and was registered in PROSPERO with registration number CRD42018091574. We graded the methodological quality of the studies using Cochrane's tool of risk of bias in nonrandomized studies-of interventions (ROBINS-I).

Results: In total, 200 studies were identified for screening, and nine studies were eligible. The quality assessment rated one study as strong, three studies (33.3\%) as fair, and five studies (55.6\%) as poor. The number of study participants ranged from 17 to 118 (total $n=394$ ), with a mean of 43.8. Four studies showed $100 \%$ clinical success with IPB. The radiographic success ranged from 60 to $100 \%$.

Conclusion: All of the included studies showed IPB treatment resulted in increased root length, progressive thickening of the dentinal walls, and narrowing of the canal space. Induced periapical bleeding is the best treatment of choice for immature permanent teeth with pulpal necrosis. Keywords: Immature permanent teeth, Necrosis pulp, Regenerative endodontic treatment, Systematic review. Conservative Dentistry and Endodontic Journal (2021): 10.5005/jp-journals-10048-0068
\end{abstract}

\section{INTRODUCTION}

Immature permanent teeth become non-vital because of infection, dental trauma, operative procedures, or congenital abnormalities. ${ }^{1}$ Current endodontic treatment involves the replacement of infected or damaged pulp with exogenous material like calcium hydroxide $(\mathrm{CH})$ or mineral trioxide aggregate (MTA). ${ }^{1-3}$ Despite its satisfactory clinical efficacy in pain elimination and infection control, they only seek to seal the space of the root canal system without restoring its original function. Therefore, it may lead to tooth fractures and reinfection of teeth. ${ }^{4,5}$ To overcome these limitations, new treatment paradigm is evolved in treatment of immature permanent teeth with necrotic pulp, which is oriented towards preservation and tissue regeneration with better understanding of biological mechanisms, the use of mesenchymal stem cells (MSCs). ${ }^{6-8}$ Induced periapical bleeding (IPB) is a regenerative treatment based on the concept that endogenous stem cells which are introduced in root canal space by lacerating the surrounding periapical tissues lead to continued root maturation, thereby increasing root length and root wall thickness and proper apical closure thereby restores the physiologically functional pulp-dentin complex., ${ }^{9,10}$

Recently many human clinical studies were conducted to show the effectiveness of IPB treatment for immature permanent teeth with necrosed pulp. ${ }^{11-28}$ The present systematic review is conducted to evaluate the quantitative effects of the IPB treatment for immature necrosed permanent teeth with an open apex.

\section{Materials and Methods}

This review was planned, conducted, and reported in adherence to PRISMA standards of quality for reporting systematic reviews and meta-analyses. ${ }^{29}$ IRB approval was not required. The present review was registered in PROSPERO with registration number CRD42018091574.
Department of Restorative Dentistry, Faculty of Dentistry, Taif University, Taif, Kingdom of Saudi Arabia

Corresponding Author: Ammar S Al Shamrani, Department of Restorative Dentistry, Faculty of Dentistry, Taif University, Taif, Kingdom of Saudi Arabia, Phone: +966554419274, e-mail: drammare@tudnet. edu.sa

How to cite this article: Al Shamrani AS. Induced Periapical Bleeding in Regeneration of Immature Permanent Teeth with Necrosis: A Systematic Review. Cons Dent Endod J 2021;6(1):6-13.

Source of support:

Conflict of interest:

\section{Questions}

We sought to examine the quantitative effects of the IPB on the healing pattern of immature permanent teeth with necrosed pulp compared to the teeth treated with other endodontic treatment procedures.

\section{Study Eligibility}

We included studies published in the English language only that investigated the effects of the IPB on the healing pattern of immature permanent teeth with necrosed pulp in humans. Papers were excluded at this stage if they were editorial letter, case report, case series, in vitro, animal studies, the regenerative endodontic procedure used for other purposes. The research question of the present systematic review was defined according to the PICO format as:

Population/Patients $(\mathrm{P})$ : Subjects with immature permanent teeth with necrosed pulp treated by IPB (MeSH-pulp treatment, endodontic treatment, pulpal therapy, permanent, teeth, dentition, pulpectomy, immature). 
Intervention (I): IPB regenerative endodontic treatment in humans only (MeSH-Regenerative, revascularization, bleeding, IPB, revitalization, induced, periapical).

Comparison (C): Treatment of immature permanent teeth with necrotic pulp by other endodontic treatment.

Outcome (O): Clinical or radiographical success with IPB regenerative endodontic treatment.

\section{Study Identification}

Various research databases were searched which include, Medline (PubMed, Ebsco, OVID Medline), Embase, (pharmacological literature, European studies), Cochrane library (Trails, Cochrane review), Web of Knowledge (Social science, conference abstract), SCOPUS (scientific web pages, conference abstracts), CINAHL (Nursing and allied health), Psyclnfo (Psychology and psychiatry), ERIC (Education) using key terms focused on the specific search strategy. For gray literature following databases were searched: Open Grey, National Library of Medicine, Google scholar, Social science research, For thesis (EthOS, DART-Europe), Institutional repositories (OpenDOAR, e-publications@RCSI Lenus, Bielefeld Base, RIAN). No beginning date was used, and the last date of the search was February 25, 2018. We searched for additional studies in the reference lists of all articles included.

\section{Study Selection and Assessment of Study Quality}

We screened all titles and abstracts independently and in duplicate for inclusion. In the event of a disagreement or insufficient information in the abstract, we independently and in duplicate reviewed the full text of potential articles. The inter-rater agreement for study inclusion, as assessed using an intra-class correlation coefficient, was 0.88 . Conflicts were resolved by consensus discussion between the two reviewers. The quality of the selected studies was assessed using the Risk of Bias tool (ROB) according to the Cochrane Handbook for Systematic Reviews of Interventions. ${ }^{30}$ The selected studies were assessed using the following criteria: random sequence generation, allocation concealment, selective reporting of outcomes, blinding of participants, personnel and outcome assessment, incomplete outcome data (reporting of dropouts), and other bias (bias due to problems not covered elsewhere in the table). Cochrane ROB tool is converted to Agency for Healthcare Research and Quality (AHRQ) standards (Good, Fair, and Poor): good quality when all criteria met (i.e., low for each domain), fair quality if one criterion not met (i.e., high risk of bias for one domain) or two criteria unclear, and the assessment that this was unlikely to have biased the outcome, and there is no known important limitation that could invalidate the results and poor quality if two or more criteria listed as high or unclear risk of bias or one criterion not met (i.e., high risk of bias for one domain) or two criteria unclear, and the assessment that this was likely to have biased the outcome, and there are important limitations that could invalidate the results.

\section{Data Extraction and Data Synthesis}

Two reviewers did data extraction independently for the included studies using a data extraction sheet, and any discrepancies were resolved by discussion and consensus. The following data were extracted from each included study: first author, publication year, study type, study quality, sample size, treated teeth, total teeth, follow-up period, method of regenerative treatment, outcome assessed, and the authors' conclusion.

\section{Results}

\section{Trail Flow}

Using our search strategy, we identified 197 articles with an additional three identified from our review of references and journal indices. From these, we identified nine articles for inclusion in the present systematic review (Flowchart 1).

\section{Study Characteristics and Study Quality}

The number of study participants ranged from 17 to 118 (total $n=394$ ), with a mean of 43.8 . Out of nine studies included in the

Flowchart 1: Study selection flow diagram

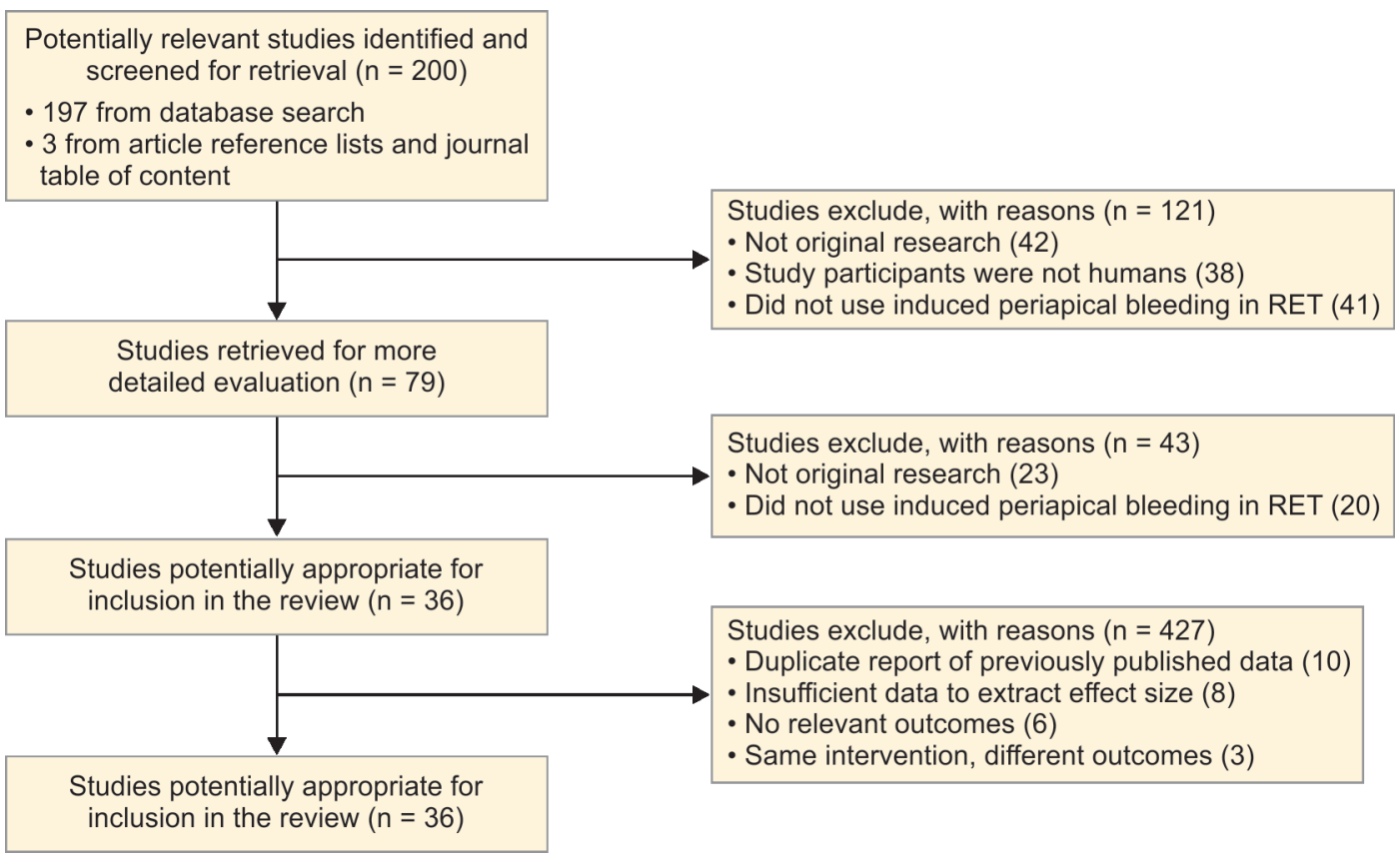


review, four studies used controls and five studies assessed the efficacy of IPB endodontic regenerative treatment on immature necrotic pulp without controls (Table 1). The follow-up period varied from 12 to 30 months (Table 2 ). In all of the included studies, sodium hypochlorite (1.5\% to $5.25 \%$ ) was used as canal irrigating agent followed by application of triple antibiotic paste. Bleeding was induced by lacerating the periapical tissues using K file (Table 3). Table 4 shows the results of the included studies. Lin et al., Li et al., Ashiry et al., and Saoud et al. showed $100 \%$ clinical success with IPB. The radiographic success with induced periapical regeneration was ranged from 60 to $100 \%$.

The quality assessment rated one study as strong, three studies (33.3\%) as fair, and five studies (55.6\%) as poor (Table 5).

\section{Discussion}

Recent treatment approaches to pulpal infection or injury to immature permanent teeth include the traditional root canal treatment with the use of improvised synthetic materials, which provide better pulpal seal compared to calcium hydroxide or biological approach based on the molecular and cellular basis for pulpal regeneration. ${ }^{2,3,11-28}$ The present systematic review was conducted to analyze the efficacy of IPB endodontic regeneration treatment on necrosed immature permanent teeth in humans. Nine studies were included in the present review. 3,12,15-19,21,22

\section{Canal Disinfection in Regeneration of Pulp}

In all of the included studies, sodium hypochlorite was used as a pulp irrigating agent, followed by the application of triple antibiotic paste for dried canals. A critical step in regenerative therapy is complete eradication of the root canal microbes using copious irrigation, placement of antibiotic pastes, and minimal instrumentation. Disinfection of pulp canal plays an important role in revascularization of immature necrotic pulp by IPB. ${ }^{31}$

\section{Method of Regenerative Procedure}

In all of the included studies, bleeding was induced into the root canals by lacerating the periapical tissues and a blood clot was allowed to form and act as a scaffold. Bleeding, in turn, delivers stem cells from the periapical area to the root canal system, and the fibrin scaffold of the blood clot entraps stem cells which are capable of initiating regeneration of necrosed pulp tissue. In all of the included studies, the coronal seal was done with MTA which in turn provided the excellent coronal sealing of pulp canal and marginal adaptation. ${ }^{6-9}$

\section{Clinical and Radiographic Outcome}

All of the included studies showed IPB regenerative treatment resulted in increased root length, progressive thickening of the dentinal walls, and narrowing of the canal space. The blood clot inside the sterile root canal acts as a matrix onto which vital periapical stem cells were seeded to reestablish pulp vascularity. These dental periapical stem cells have tissue regeneration potential and proliferated into the newly formed blood clot matrix, differentiate into odontoblasts, and deposit tertiary or tubular dentin. Because of its apical location, the apical papilla has a collateral circulation that enables it to survive during the process of pulp necrosis. ${ }^{6-9}$ Lin et al. ${ }^{3}$ compared IPB with calcium hydroxide apexification and showed a significant increase in root length, canal wall thickness, and closure of apical foramen with RET group compared to controls. Nagy et al. ${ }^{22}$ compared IPB alone

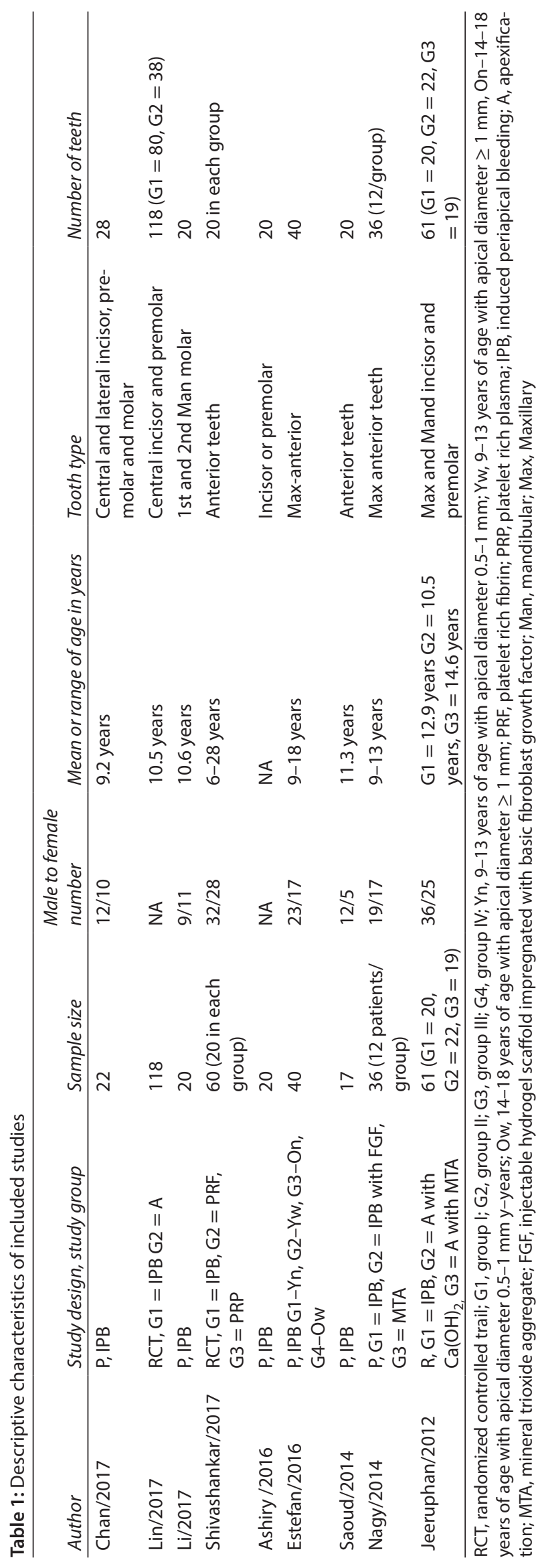




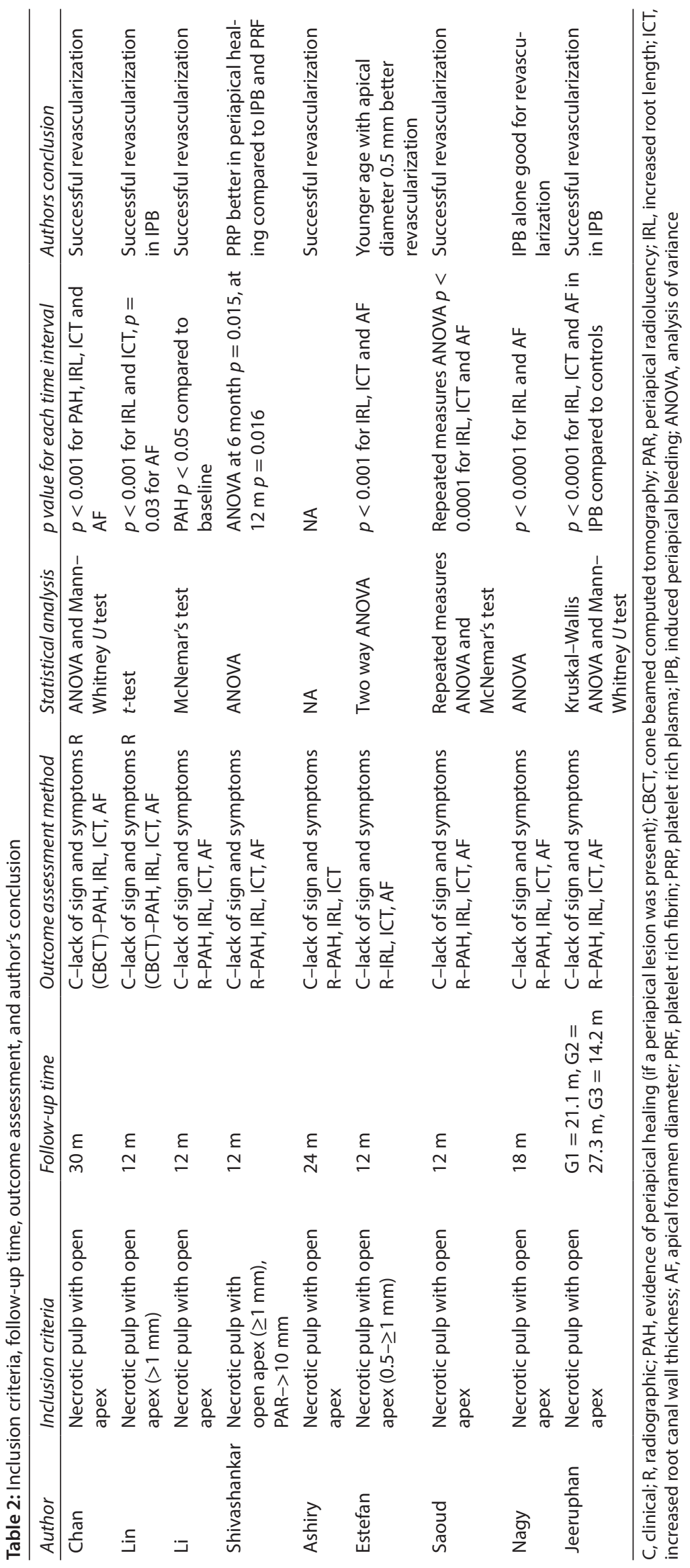




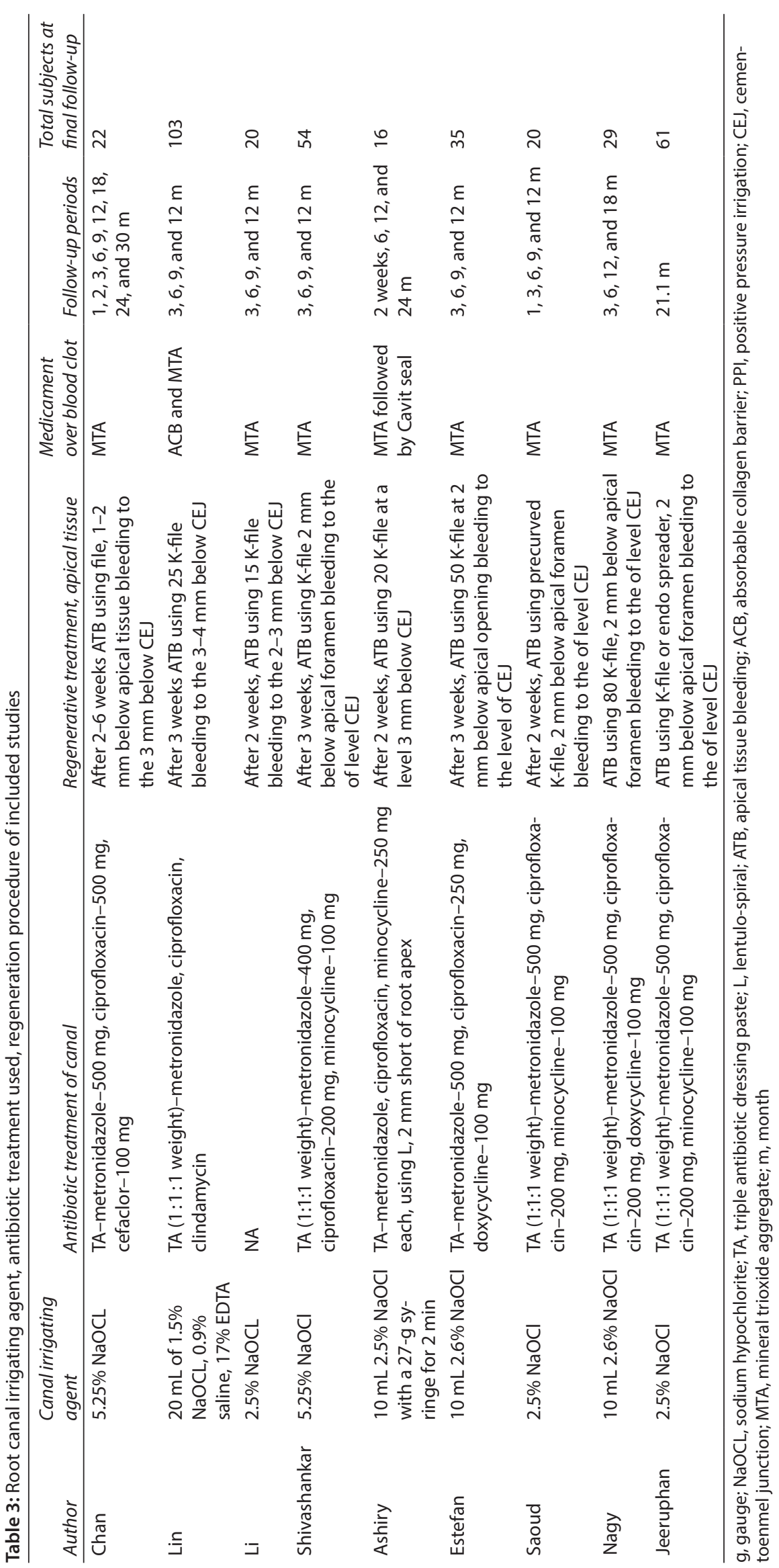




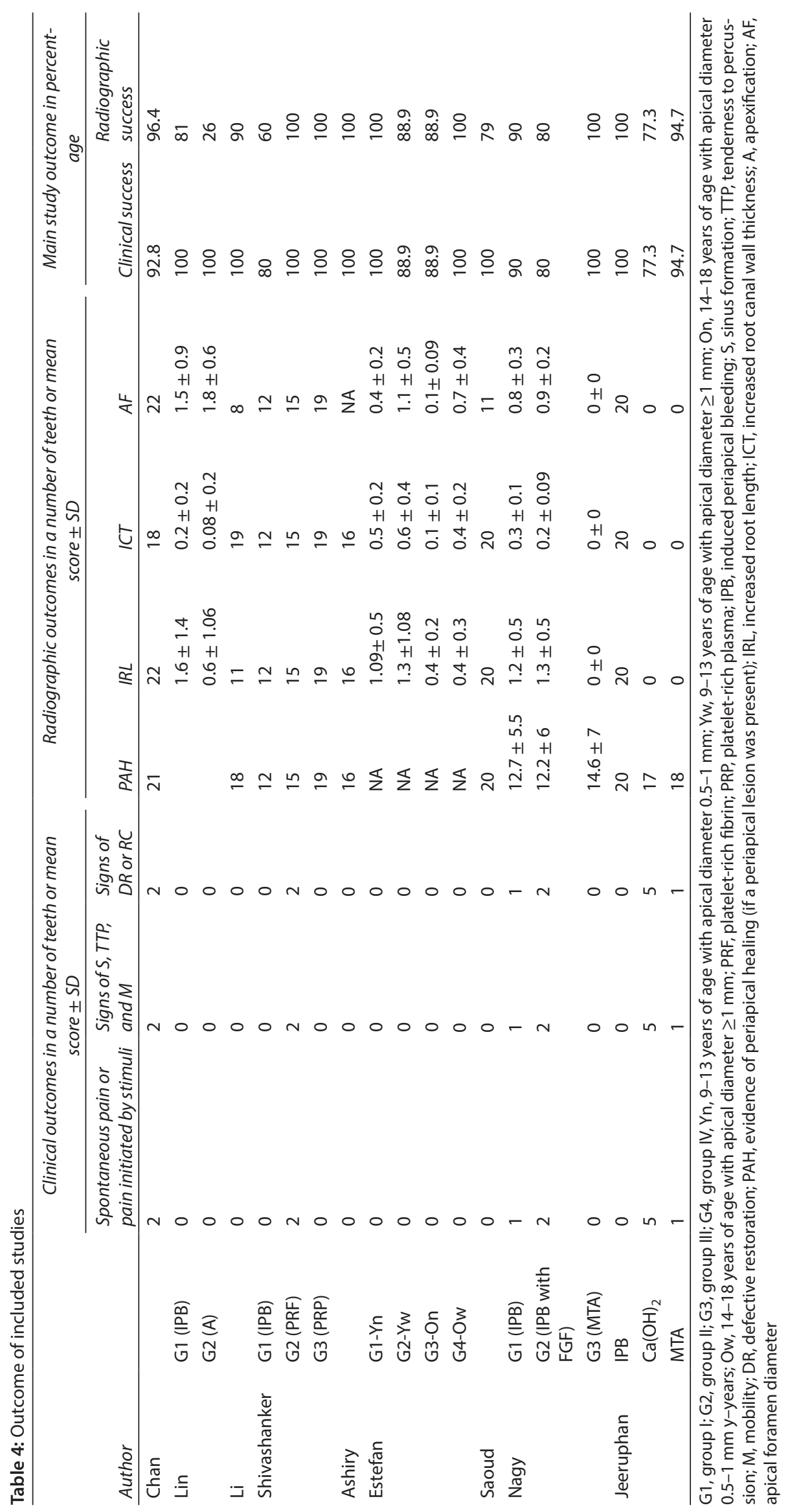


Induced Periapical Bleeding in Pulp Regeneration

Table 5: Risk of bias tool for included studies

\begin{tabular}{llllllll}
\hline & \multicolumn{7}{c}{ Risk of bias tool } \\
\cline { 2 - 6 } Author/year & RSG & AC & BPPO & IOD & SOR & OSB & AHRQ rating \\
\hline Chan/2017 & Low & Low & High & Low & Low & Low & Fair \\
Lin/2017 & High & Low & High & Low & Low & Low & Poor \\
Li/2017 & High & Unclear & Low & High & Unclear & Low & Poor \\
Shivashankar/2017 & Low & Low & Low & Low & Low & Low & Good \\
Ashiry/2016 & High & High & Low & High & High & Unclear & Poor \\
Estefan/2016 & High & High & Low & High & Low & Unclear & Poor \\
Saoud/2014 & High & Unclear & Low & Low & Low & Low & Fair \\
Nagy/2014 & High & Unclear & Low & Low & Low & Low & Fair \\
Jeeruphan/2012 & High & Low & Unclear & Low & High & Low & Poor \\
\hline
\end{tabular}

RSG, random sequence generation; AC, allocation concealment; BPPO, blinding of participants, personnel and outcome assessors; IOD, incomplete outcome data; SOR, selective outcome reporting; OSB, other sources of bias; $A H R Q$, agency for healthcare research and quality

and IPB with fibroblast growth factors (FGFs) with MTA. The result showed significant radiographic changes in IPB and IPB with the FGF group compared to MTA.

\section{Study Limitations}

The limitation of the present review was, due to heterogeneity across the studies, we could not do the meta-analysis of included studies. The heterogeneity was caused by variation in the in vivo models, such as follow-up period, type of teeth included, age of the study participants. Therefore, no forest plots or funnel plots were constructed. Due to the disparate nature of the studies, only simple descriptive and stratified comparisons are reported. The quality assessment rated only one study as good, three as fair, and four as poor. The present results point to the need for high-quality randomized controlled trials in further research and the need for alternative methods to perform "in vitro" researches on the effects of pulp regenerations in customized models.

\section{CONCLUSION}

All of the included studies showed IPB regenerative endodontic treatment resulted in increased root length, progressive thickening of the dentinal walls, and narrowing of the canal space. Induced periapical bleeding is the best treatment of choice for immature permanent teeth with pulpal necrosis.

\section{Acknowledgments}

Thankful to Dr Roshan Noor Mohamed and Dr Sakeenabi Basha for helping in statistical analysis and editing of the data.

\section{References}

1. Flanagan TA. What can cause the pulps of immature, permanent teeth with open apices to become necrotic and what treatment options are available for these teeth. Aust Endod J 2014;40(3):95-100. DOI: 10.1111/aej.12087.

2. Agrafioti A, Giannakoulas DG, Filippatos CG, et al. Analysis of clinical studies related to apexification techniques. Eur J Paediatr Dent 2017;18(4):273-284. DOI: 10.23804/ejpd.2017.18.04.03.

3. Lin JC, Lu JX, Zeng Q, et al. Comparison of mineral trioxide aggregate and calcium hydroxide for apexification of immature permanent teeth: a systematic review and meta-analysis. J Formos Med Assoc 2016;115(7):523-530. DOI: 10.1016/j.jfma.2016.01.010.

4. Belli S, Eraslan O, Eskitaşcıoglu G. Effect of different treatment options on biomechanics of immature teeth: a finite element stress analysis study. J Endod 2018;44(3):475-479. DOI: 10.1016/j.joen.2017.08.037.
5. Demiriz L, Bodrumlu EH. Severe unintentional extrusion of mineral trioxide aggregate during apexification. Indian J Dent Res 2017;28(3):341-343. DOI: 10.4103/ijdr.IJDR_365_16.

6. Anitua E, Sanchez M, Orive G. Potential of endogenous regenerative technology for in situ regenerative medicine. Adv Drug Deliv Rev 2010;15(62):741-752. DOI: 10.1016/j.addr.2010.01.001.

7. Mao JJ, Kim SG, Zhou J, et al. Regenerative endodontics: barriers and strategies for clinical translation. Dent Clin North Am 2012;56(3):639649. DOI: 10.1016/j.cden.2012.05.005.

8. Huang GT. Pulp and dentin tissue engineering and regeneration: current progress. Regen Med 2009;4(5):697-707. DOI: 10.2217/ rme.09.45.

9. Bezgin T, Sonmez H. Review of current concepts of revascularization/ revitalization. Dent Traumatol 2015;31(4):267-273. DOI: 10.1111/ edt.12177.

10. Hilkens $\mathbf{P}$, Meschi $\mathrm{N}$, Lambrechts $\mathrm{P}$, et al. Dental stem cells in pulp regeneration: near future or long road ahead? Stem Cells Dev 2015;24(14):1610-1622. DOI: 10.1089/scd.2014.0510.

11. Alobaid AS, Cortes LM, Lo J, et al. Radiographic and clinical outcomes of the treatment of immature permanent teeth by revascularization or apexification: a pilot retrospective cohort study. J Endod 2014;40(8):1063-1070. DOI: 10.1016/j.joen.2014.02.016.

12. Shivashankar VY, Johns DA, Maroli RK, et al. Comparison of the effect of PRP, PRF and induced bleeding in the revascularization of teeth with necrotic pulp and open apex: a triple blind randomized clinical trial. J Clin Diagn Res 2017;11(6):ZC34-ZC39. DOI: 10.7860/ JCDR/2017/22352.10056.

13. Silujjai J, Linsuwanont P. Treatment outcomes of apexification or revascularization in nonvital immature permanent teeth: a retrospective study. J Endod 2017;43(2):238-245. DOI: 10.1016/j. joen.2016.10.030.

14. Bukhari S, Kohli MR, Setzer F, et al. Outcome of revascularization procedure: a retrospective case series. J Endod 2016;42(12):1752-1759. DOI: 10.1016/j.joen.2016.06.021.

15. Chan EK, Desmeules M, Cielecki M, et al. . Longitudinal cohort study of regenerative endodontic treatment for immature necrotic permanent teeth. J Endod 2017;43(3):395-400. DOI: 10.1016/j. joen.2016.10.035.

16. Li L, Pan Y, Mei L, et al. Clinical and radiographic outcomes in immature permanent necrotic evaginated teeth treated with regenerative endodontic procedures. J Endod 2017;43(2):246-251. DOI: 10.1016/j. joen.2016.10.015.

17. Estefan BS, El Batouty KM, Nagy MM, et al. Influence of age and apical diameter on the success of endodontic regeneration procedures. J Endod 2016;42(11):1620-1625. DOI: 10.1016/j.joen.2016.06.020.

18. Saoud TM, Zaazou A, Nabil A, et al. Clinical and radiographic outcomes of traumatized immature permanent necrotic teeth after revascularization/revitalization therapy. J Endod 2014;40(12):19461952. DOI: 10.1016/j.joen.2014.08.023. 
19. Jeeruphan T, Jantarat J, Yanpiset K, et al. Mahidol study 1: comparison of radiographic and survival outcomes of immature teeth treated with either regenerative endodontic or apexification methods: a retrospective study. J Endod 2012;38(10):1330-1336. DOI: 10.1016/j. joen.2012.06.028.

20. Bose R, Nummikoski P, Hargreaves K. A retrospective evaluation of radiographic outcomes in immature teeth with necrotic root canal systems treated with regenerative endodontic procedures. J Endod 2009;35(10):1343-1349. DOI: 10.1016/j.joen.2009.06.021.

21. El Ashiry EA, Farsi NM, Abuzeid ST, et al. Dental pulp revascularization of necrotic permanent teeth with immature apices. J Clin Pediatr Dent 2016;40(5):361-366. DOI: 10.17796/1053-4628-40.5.361.

22. Nagy MM, Tawfik HE, Hashem AA, et al. Regenerative potential of immature permanent teeth with necrotic pulps after different regenerative protocols. J Endod 2014;40(2):192-198. DOI: 10.1016/j. joen.2013.10.027.

23. Ding RY, Cheung GS, Chen J, et al. Pulp revascularization of immature teeth with apical periodontitis: a clinical study. J Endod 2009;35(5):745-749. DOI: 10.1016/j.joen.2009.02.009.

24. Chen X, Bao ZF, Liu Y, et al. Regenerative endodontic treatment of an immature permanent tooth at an early stage of root development: a case report. J Endod 2013;39(5):719-722. DOI: 10.1016/j.joen.2012.12.023.
25. Chueh LH, Ho YC, Kuo TC, et al. Regenerative endodontic treatment for necrotic immature permanent teeth. J Endod 2009;35(2):160-164. DOI: 10.1016/j.joen.2008.10.019.

26. Wigler R, Kaufman AY, Lin S, et al. Revascularization: a treatment for permanent teeth with necrotic pulp and incomplete root development. J Endod 2013;39(3):319-326. DOI: 10.1016/j. joen.2012.11.014

27. Kahler B, Mistry S, Moule A, et al. Revascularization outcomes: a prospective analysis of 16 consecutive cases. J Endod 2014;40(3):333338. DOI: 10.1016/j.joen.2013.10.032.

28. Tawfik H, Abu-Seida AM, Hashem AA, et al. Regenerative potential following revascularization of immature permanent teeth with necroticpulps. Int Endod J 2013;46(10):910-922. DOI: 10.1111/iej.12079.

29. Moher D, Liberati A, Tetzlaff J, et al. Preferred reporting items for systematic reviews and meta-analyses: the PRISMAstatement. J Clin Epidemiol 2009;62(10):1006-1012. DOI: 10.1016/j.jclinepi.2009.06.005.

30. Sterne JA, Hernán MA, Reeves BC. ROBINS-I: a tool for assessing risk of bias in non-randomised studies of interventions. BMJ 2016;355:14919 Albuquerque MTP, Nagata J, Bottino MC, et al. Antimicrobial efficacy of triple antibiotic-eluting polymer nanofibers against multispecies biofilm. J Endod 2017;43(9S):S51-S56. DOI: 10.1136/bmj.i4919.

31. Albuquerque MT, Valera MC, Nakashima M, et al. Tissue-engineeringbased strategies for regenerative endodontics. J Dent Res 2014;93(12):1222-1231. DOI: 10.1177/0022034514549809. 